\title{
Low-cost Differential GPS for Field Robotics
}

\author{
Ben Nizette ${ }^{1}$, Andrew Tridgell ${ }^{2}$ and Changbin $\mathrm{Yu}^{1}$
}

\begin{abstract}
In this paper we detail the design and implementation of a Differential GPS system that uses only low-cost, light-weight and widely available GPS modules. Such a system is desirable for modern field robotics in order to improve the performance of mapping, navigation, collision avoidance and other common tasks, especially on payload-constrained robots. The particular noise and error modes present in the low-cost system are investigated and algorithms introduced to minimise their effect. The system is demonstrated in the context of field robotics for stationary, ground-based and airbourne agents. It is found that the majority of the error due to the low cost components can be manipulated to be of a form easily mitigated for most robots. The system is shown to provide good results in the horizontal plane in open environments such as those typically used for outdoor experimental robotics while being one to two orders of magnitude cheaper than comparable commercial systems.
\end{abstract}

\section{INTRODUCTION}

Precise knowledge of the location of an agent is a vital piece of information in many field robotic tasks. Localisation of a single agent is required for mapping, navigation and automated interaction with the environment. With expansion in the field of multi-agent systems, high quality localisation has become even more important in order to cover tasks such as formation control and collision avoidance.

Global Navigation Satellite Systems (GNSS), of which GPS is the most prominent example, are very widely used in field robotics to provide localisation. Simple GPS has an accuracy of between 3 and 10 metres[1] depending on environmental factors and the availability of services such as SBAS. As such, it does not necessarily provide the accuracy required for high quality mapping, small to medium scale formation control and collision avoidance and other similar tasks.

Researchers have historically sought to improve results from simple GPS units by using some knowledge of the agent's dynamic model through Kalman filtering[2]. This can be extended to fuse data from other sensors such as odometry[3] or laser rangers[4]. Improvement in the relative accuracy of the agent's path can also be found by fusing the GPS data with that from an inertial measurement unit (IMU)[5], [6] however this cannot in general remove biases or offsets in the position solution with respect to the global frame.

\footnotetext{
${ }^{1}$ Ben Nizette and Changbin $\mathrm{Yu}$ are with the Research School of Engineering, Australian National University (ANU), Canberra, Australia. benjamin.nizettedanu.edu.au, brad. yueanu. edu. au

${ }^{2}$ Andrew Tridgell is with the Research School of Computer Science, Australian National University (ANU), Canberra, Australia. andrewatridgell. net
}

Higher quality GPS localisation is available by using methods such as Differential or Real-Time Kinematic GPS (DGPS, RTK). Each of these methods require a stationary base in the vacinity of the agents with at least a unidirectional telemetry link for the broadcast of corrections. The cost and size of these solutions limits their use to small numbers of large agents, typically agricultrual machinery[7], [8], selfdriving cars[9], [4] or other large robots[3].

Open-source packages such as RTKLIB[10] provide implementations of several RTK and DGPS algorithms. In order to use RTKLIB in field robotic scenarios, a tradeoff must be made between adding computational hardware on board the robot, or introducing latencies by centralised computation of the RTK/DGPS algorithms. Moreover, these software packages do not provide a low-latency GPS velocity estimate, something that is required for accurate attitude estimation in some avionics systems[11]. This may in turn require the overhead of two separate GPS modules being used on the robot.

Recently, low-cost GPS modules have become available that are capable of receiving DGPS corrections. This lowers the complexity and cost of an implementing agent as it need only be equipped with this standard GPS module a radio capable of receiving that data. An example of such a module is the uBlox LEA family, widely used in robotics and available for around AU\$30 a piece. A DGPS base station is still required in this scenario and commercial units are typically several hundreds to thousands of dollars, once again, limiting application.

In this paper we detail the design and implementation of a DGPS base station that uses only a cheap GPS module and a small Linux computer, available together for approximately AU\$100. The particular accuracy and error modes of this set up are analysed and its suitability is examined for common field robotic tasks. It is assumed that the agents either have no strong requirement for altitude data (such as ground vehicles) or have a barometric pressure sensor or similar device that may simply be fused with the GPS data for refinement of the vehicle height. The developed GPS system is thus required to have higher accuracy in the horizontal plane than along the height axis. The target absolute accuracy in this plane is in the order of one metre; this is sufficient for small- to medium-scale mapping, navigation and collision avoidance tasks and is comparable to the performance of commercial DGPS solutions.

The data and calculations required to generate the DGPS corrections are detailed in Section II for both the developed DGPS station and third-party sources of correction used for comparison. The hardware used is described in Section III 
followed by the results of experiments in urban and rural environments in Section IV. Potential for future work is given in Section V.

\section{Correction Calculation}

The GPS system is comprised of a series of Space Vehicles (SVs) orbiting the Earth, each containing a high accuracy clock. The SVs transmit known pseudorandom sequences that are picked up by GPS receivers on the Earth. These receivers may correlate the received sequences against the known standards to determine the transmission time and from that, the pseudorange to the satellite. The term pseudorange reflects the fact that the receiver's clock is not synchronised with the SVs so the measured values contain a component due to both the spacial displacement of the devices and also the difference in their clocks.

The signals propagate to the receiver through the Earth's atmosphere which affects the propagation speed and therefore apparent range to the SV. This error is the major nondeterministic contributor to errors in GPS localisation[1]. It is also strongly correlated between nearby receivers - simply, if two receivers are near each other then the SV signals to each will have travelled through the same portion of the atmosphere and therefore should be similarly corrupted. If one receiver knows its location a-priori then it may observe the GPS signals and work backwards to deduce the error introduced by the atmosphere. This error can be sent to nearby roving receivers which may then subtract it from their observations before performing their localisation. This is termed Differential GPS (DGPS).

The DGPS base station will also observe errors that are not necessarily spacially correlated with nearby receivers and will incorrectly report these to the rovers for removal. Such local errors include receiver clock error, local multipath effects. A DGPS system only offers an improvement in position for the rovers if these incorrectly reported local errors are much smaller in magnitude than the correctly reported common-mode atmospheric effects. This is the traditional motivation for expensive DGPS reference stations - high quality clocks and antennae that minimize local error. The key question to be answered when designing a cheap DGPS reference is what form the increased magnitude of the local errors take due to low quality clocks and antennae, and how their action at the rovers may be mitigated.

\section{A. Local Corrections}

The implementation of the stationary DGPS reference is typically a GPS receiver with connection to a small computer (possibly embedded in the GPS module). This reference receiver front-end must have a known position in space and be capable of exposing the raw pseudorange and carrier phase measurements to the computer such that the computer may calculate the per-satellite errors.

The pseudorange measurements are considered the primary data when performing a GPS positional solution; they encode the distance information from each satellite to the receiver in four dimensions (three spacial plus time).
Measurements of the underlying carrier phase provide a high accuracy distance measurement, but are subject to an integer ambiguity in the number of wavelengths between receiver and satellite and as such cannot be used directly. The use of carrier phase for localization by means of integer ambiguity resolution is discussed in, for example [5], [12]. For this application, the phase information was integrated with the pseudorange in the form of a Hatch fitler[13] which provides a smoothing of the pseudorange using the difference in observed carrier phase between successive measurements and is not subject to integer ambiguity.

The position in space of the reference receiver is assumed known, however the reference receiver clock error relative to the GPS time is not known. This parameter is required to be found from the raw measurements.

The smoothed pseudoranges were corrected for known sources of error according to

\begin{tabular}{|c|c|}
\hline \multirow{2}{*}{\multicolumn{2}{|c|}{$\begin{array}{r}\rho \\
\text { Where }\end{array}$}} \\
\hline & \\
\hline$\rho$ & Corrected Pseudorange \\
\hline$\rho_{0}$ & Raw Pseudorange \\
\hline$r_{\text {rel }}$ & Relativistic correction \\
\hline$r_{\text {rot }}$ & Correction for Earth's rotation \\
\hline$T_{\mathrm{sv}}$ & Satellite clock error \\
\hline$T_{\mathrm{gd}}$ & Satellite group delay \\
\hline$c$ & Speed of light \\
\hline
\end{tabular}

Weightings are defined on each observation according to the quality of the signal from each satellite, that satellite's elevation and how long the Hatch filter history length is (and therefore the quality of pseudorange smoothing). For the $i^{\text {th }}$ satellite

$$
w_{i}=\frac{1}{(8-q)^{2}} \frac{\min \left(e l_{i}, e l_{\max }\right)}{e l_{\max }} \frac{\min \left(N_{i}, N_{\max }\right)}{N_{\max }}
$$

With $N$ the number of consistent samples in the Hatch filter history, $q$ the quality metric from GPS Front End (17) and $e l_{\max }$ and $N_{\max }$ tuning parameters set to $10^{\circ}$ and 20 measurements respectively.

The standard pseudorange equations are linear in the receiver clock error $T_{\mathrm{rx}}$ so we may easily estimate this parameter. Denoting the estimate of receiver clock error as $\hat{T}_{\mathrm{rx}}$, receiver position $\left[\begin{array}{lll}x & y & z\end{array}\right]^{\mathrm{T}}$, noise $\epsilon$ and a subscript $i$ denoting a variable specific to the $i^{\text {th }}$ satellite

$$
\begin{aligned}
\rho_{i} & =\sqrt{\left(x_{i}-x\right)^{2}+\left(y_{i}-y\right)^{2}+\left(z_{i}-z\right)^{2}}+c T_{\mathrm{rx}}+\epsilon_{i} \\
& =C_{i}+c T_{\mathrm{rx}}+\epsilon_{i} \\
\text { so } & \\
\hat{T}_{\mathrm{rx}} & =\frac{1}{c} \frac{\sum_{i}^{n} w_{i}\left(\rho_{i}-C_{i}\right)}{\sum_{i}^{n} w_{i}}
\end{aligned}
$$

With the receiver clock error thus approximated, the observed pseudoranges at the reference receiver were corrected 
to obtain approximate observed geometric ranges to each of the satellites then differenced with the true geometric distance. The correction measurement $y$ is then:

$$
y=\rho+c \hat{T}_{\mathrm{rx}}-r_{\mathrm{geo}}
$$

The true geometric distance $r_{\text {geo }}$ is found by extracting each satellite's position from the broadcast Ephemeris and differencing with the reference's known location.

These corrections were generated at the receiver output frequency of $5 \mathrm{~Hz}$ and stored in a time-sorted buffer of configurable length. At each output time step the history buffer was sorted by correction magnitude and the output given as the mean of the centre half of this magnitude-sorted buffer. That is, for time sorted First-in First-out history buffer $y_{1 \ldots n}$ and correction signal $\Delta \rho$ :

$$
\begin{aligned}
m_{1 \ldots n} & =\operatorname{sorted}\left(y_{i \ldots n}\right) \\
\Delta \rho & =\sum_{i=n / 4}^{3 n / 4} m_{i}
\end{aligned}
$$

This was effective in rejecting several-sample outliers without adversely affecting the bandwidth of the correction signal.

Remark Equation 5 is a design choice that was found to be effective in practice. Statistical analysis of large data sets may yeild an optimal form of this equation and will be discussed in future work.

The output format for the correction data was chosen to be RTCMv2 in order to be compatible with the chosen lowcost receivers. This standard requires that the corrections as transmitted also include the satellite clock errors, group delay and relativistic correction; each calculated according to the GPS Interface Control Document[14].

The RTCMv2 format also requires an estimate of the rate of change of each correction in order to extend the useful life of the corrections in the receiver[15]. This rate was calculated simply as the first order difference of preceeding samples.

The User Differential Range Error estimate (UDRE) field of the RTCMv2 message provides an opportunity for the reference station to communicate information to the receivers regarding the certainty of the error for each particular satellite. This allows the receiver to adjust the satellite weightings in their internal least-squares position solution. For this system, the UDRE used for each satellite was exactly the weighting factor given in Equation 2

\section{B. Infrastructure Corrections}

The system was extended to receive correction information from external Continuously Operating Reference Stations (CORS) operated by a third party. These stations provide signal observations in RTCMv3 format [16] encapsulated in NTRIP for internet transport.

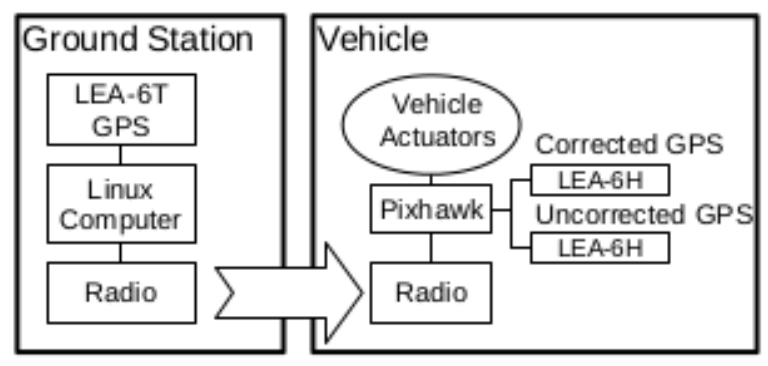

Fig. 1. System diagram

The RTCMv3 Observation messages required translation to RTCMv2 Correction messages understood by the receivers. The Observation messages contain similar pseudorange and carrier phase measurements as were received from the GPS receiver in the locally-generated system of the previous section. The difference being that the Observation messages from the CORS had already been corrected for the reference station's receiver clock error, meaning that a further least-squares estimation of this parameter was not necessary. Once the RTCMv3 Observations were then unpacked from the NTRIP transport, they were injected into the same infrastructure as used for the local corrections at a point just after the local corrections had performed their receiver clock error estimation.

\section{HARDWARE DESIGN}

Each agent in the system was equipped with two lowcost uBlox LEA-6H GPS modules[17] with integrated $25 \mathrm{~mm}$ patch antennas. These modules were chosen as they are already widely used in the hobbyist and research communities and have the option to receive RTCMv2 differential GPS corrections. The GPS modules were connected to Pixhawk avionics running Ardupilot autopilot software along with a radio telemetry system. It should be noted that the perrover set up of this DGPS system is identical to what would be required for a non-corrected GPS system. The Pixhawk/Ardupilot system is flexible enough to be used to control ground-rover agents as well as aerial agents; both such agent types are examined in the sequel.

Only one of the two modules on each rover was corrected but both outputs were recorded, allowing a control signal against which to verify the effect of the differential corrections.

A MAVLink message was created allowing the transport of RTCMv2 data from the ground station to the GPS via the avionics telemetry channel. The ground station was scripted as a MAVProxy instance with a module listening for RTCMv2 data on a UDP port, allowing the ground station and GPS reference station to be physically separate.

The reference station was chosen to be a low-cost Linux computer interfaced with a raw-data capable uBlox LEA6T GPS module with a remote $25 \mathrm{~mm}$ active patch antenna. This same reference station optionally could be connected to an NTRIP server via the internet for the receipt of 
RTCMv3 observations from a third-party Continually Operating Reference Station. The proposed algorithm was found to use a negligable portion of the resources available on even these low-cost computers, no performance advantage is expected if the system were to be implemented on more expensive computational hardware. The uBlox family of rawcapable receivers were chosen for their availability, low cost and communications compatibility with the roving receivers. The use of other low-cost raw-capable GPS receivers such as the NVS Techologies NV08C-CSM is possible but not investigated in this work.

All code is released under the terms of Version 3 of the GNU Public Licence at http://github.com/benizl/ pyUblox.

\section{RESUlTS}

All experiments had an RTCMv2 update rate of $1 \mathrm{~Hz}$, downsampled from the reference station solution rate of $5 \mathrm{~Hz}$. This was found to offer the best compromise between DGPS performance and telemetry bandwidth.

Several days of recorded raw data was examined for outliers; periods where the correction signal for any satellite deviated from the expected value by more than 5 metres. This is an indication that the GPS has lost lock on the satellite, has incorrectly chosen a multipath signal or otherwise is not providing healthy data from that satellite. It was found that these periods typically lasted less than three seconds and should form no more than one quarter of the history buffer length of Equation 5 in order to be effectively rejected. As such, the history buffer length was set to 15 seconds for all experiments.

\section{A. Reference Position Discovery}

For each new experimental environment, the ground truth position of the reference receiver had to be discovered and recorded. In each case, the reference receiver was left to record raw observations for a 72-hour period. At the completion of this time, the observations were converted to RINEX format and uploaded to the Canadian Spacial Reference System's Precise Point Positioning service[18]. This is a free service that provides a high quality postprocessed GPS localization solution of user observations incorporating atmospheric observations recorded at the time of the user's observations along with high rate ephemeris data from the measured orbits of the satellites and as such, it can provide much more accurate localization than a realtime service.

All experiments had the GPS module's internal dynamic model set to the most permissive setting "Airbourne 4G". This was done in order to minimise the filtering performed internally on position and as such to get the clearest picture of the effect of the differential corrections by themselves.

\section{B. Urban Environments}

The first experiment was set up in an urban environment, on the roof of a dwelling. The three modules (reference, corrected and uncorrected receivers) were colocated in order

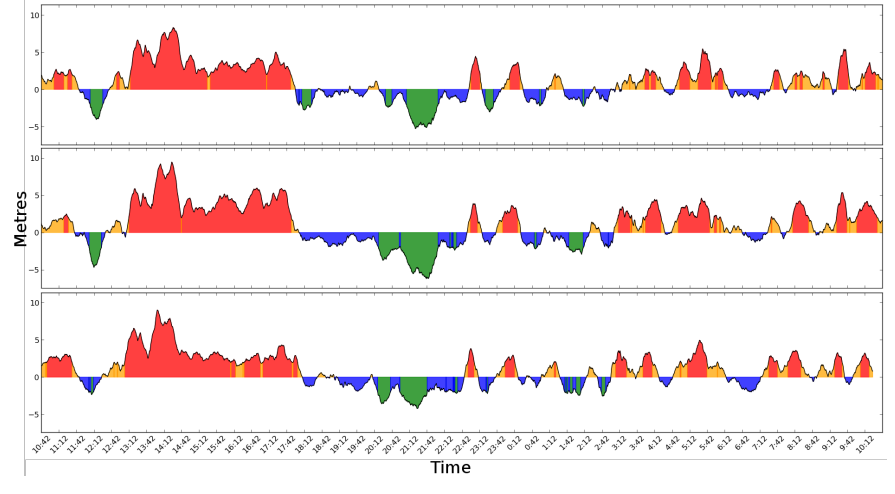

Fig. 2. 72 hour capture of relative improvement of DGPS performance in high multipath environment(metres). Positive is degredation of performance, negative is improvement.

to simplify truthing. The system was run for three days and the distance from each of the roving receivers' reported positions to their true position recorded.

The difference between these two distances was used as a metric for the performance of the system; if the correction brought the solution significantly closer to truth than without then the system was deemed to be performing as intended.

This difference is plotted over time in Figure 2 with negative numbers indicating an improvement in performance.

Observation The addition of the corrections in this environment made the position significantly worse for much of the operational period. Moreover, this performance was strongly correlated with the time of day, indicating that it was caused by a property of the satellite consellation (which also repeats every 24 hours[5]).

Claim The tight correlation of the correction quality with the satellite constellation indicates that the corrected received is more strongly influenced by multipath signals from adjoining buildings as compared to the uncorrected receiver. It is concluded that the cheap GPS modules used disable some amount of internal weighting heuristic when supplied with external corrections.

It is not feasible to determine whether the rover is seeing multipath and adjust the input UDRE weights from there as the roving receivers do not provide raw outputs. Moreover this would not scale to large numbers of rovers as each one would require a customised RTCMv2 message.

As such it is concluded that the proposed differential GPS system is not suitable for high multipath urban environments due to proportionally-greater and unavoidable influence of multipath signals in the corrected receiver relative to an uncorrected module.

\section{Field Environments}

An experimental facility was then set up in a field location. No significant obstructions were present in the vacinity of the equipment so few multipath signals existed. 


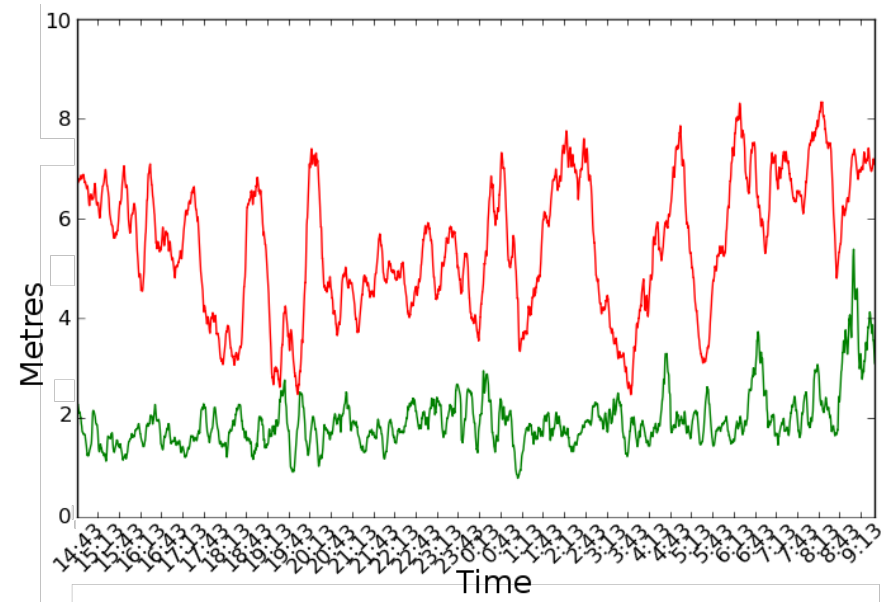

Fig. 3. Absolute accuracy with respect to ground truth, corrected (green) and uncorrected (red) receivers

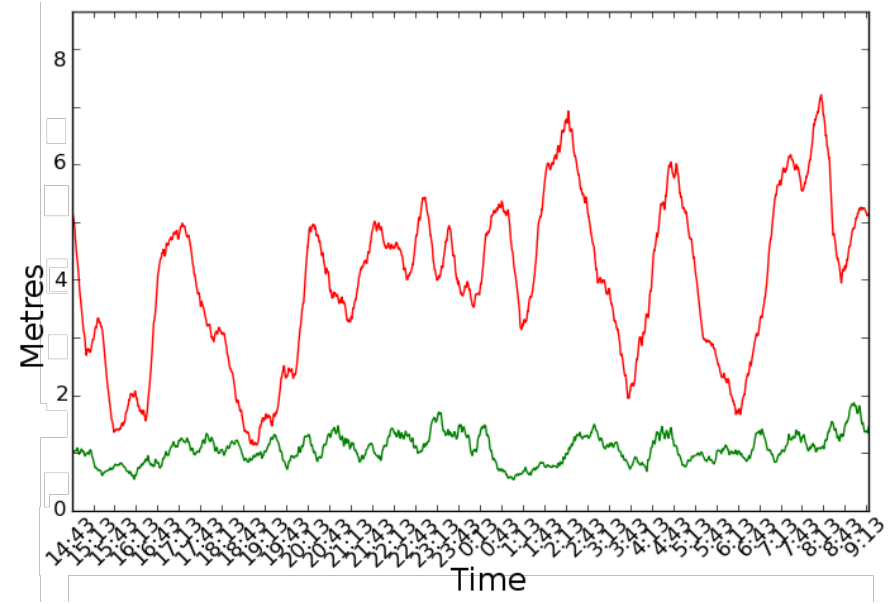

Fig. 4. Absolute accuracy with respect to ground truth, East/North plane only, corrected (green) and uncorrected (red) receivers.

Similarly to the urban set up, three receivers were colocated with one providing corrections, one receiving corrections and one uncorrected control receiver.

Observation The corrected receiver showed significantly better performance than the uncorrected with respect to ground truth. In three dimensions, the error was on the order of two metres (Figure 3) while also showing more consistant positions over time.

In the East/North plane, the receiver showed an error on the order of one metre with respect to ground truth.

Claim Accuracy in the EN plane is the most important for field robotics due to the ready availability of height data from other sources. The demonstrated accuracy in the horizontal plane meets the design goal as the order of one metre

\section{Ground Vehicle Testing}

Testing was then performed using a ground rover robot. The robot was equipped with two GPS modules as before, one corrected and one uncorrected, both connected to Pixhawk avionics running the ArduRover code. A course was

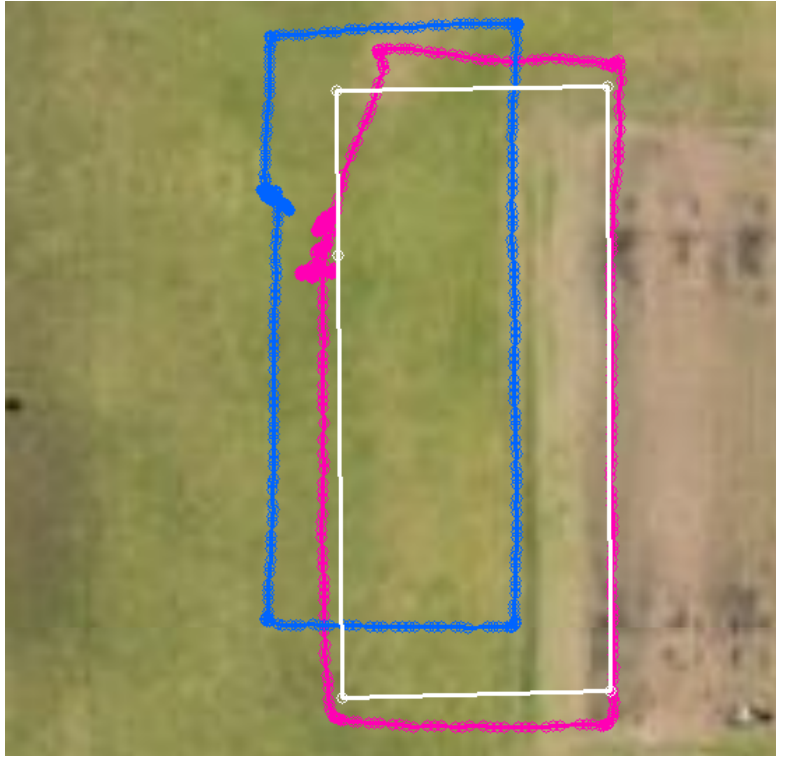

Fig. 5. GPS path of corrected (purple) and uncorrected (blue) receivers with ground truth path (white)

surveyed out on the ground and the rover was moved around that course. The plot of corrected and uncorrected GPS paths along with the surveyed ground truth can be seen in Figure 5.

Observation The corrected GPS receiver performs significantly better with respect to ground truth location. The accuracy of the corrected track shape is not as good as the uncorrected receiver. Moveover, the accuracy of the shape in the North/South axis is worse than in the East/West

Remark The corrected receiver is affected by two sources of noise: It's own and that at the reference receiver. This extra random noise manifests as greater short-term deviations from the true path, most visible by a softening of the corners of the track.

Claim The accuracy difference between North and East axes indicates that a common offset was present in all corrections at any given time. Given the geometry of the GPS constellation and the lattitude of the test site, this constant offset would lead on average to a greater error contribution along that North/South axis. More error again would be expected in altitude, however ground truth altitude data was not available for the test site, nor is it particularly relevant to field robotics (as discussed above).

This is expected to be due to the receiver clock error which is common to all rovers receiving corrections from any one reference station. It is not expected to contribute significantly to error in relative position between agents.

\section{E. Airbourne Testing}

A critical property of GPS modules for aerial robotics is their path stability at speed and during high-acceleration manouvres. A test was run with corrected and uncorrected receivers fitted to Pixhawk avionics and placed inside a fixed- 


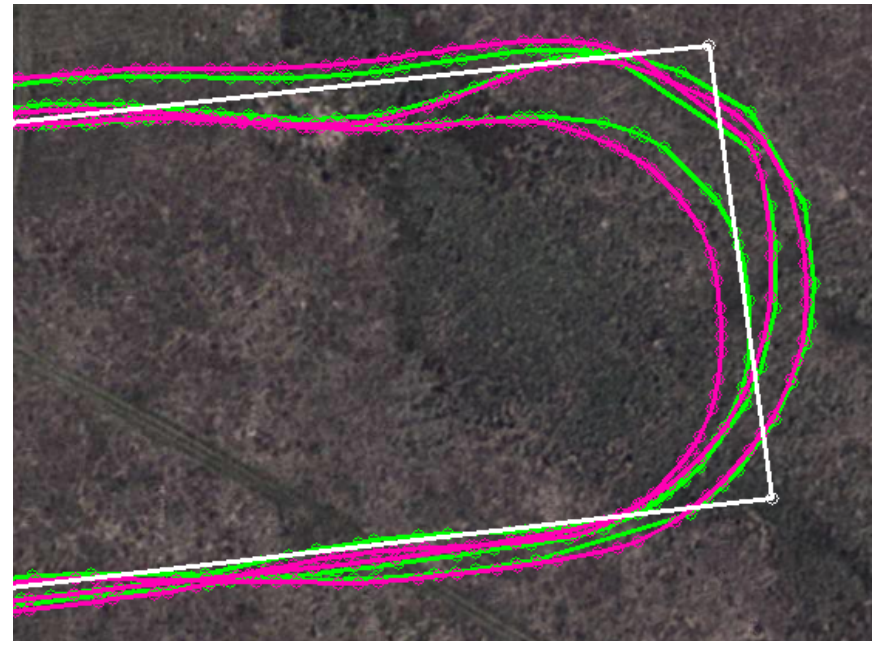

Fig. 6. GPS path of corrected (purple) and uncorrected (green) receivers with waypoint path (white)

wing aircraft. The purpose of the test was to ensure that there was no significant degredation of the path performance of the corrected module at high speed and/or high acceleration.

Flight paths during a coordinated turn through two waypoints can be seen in Figure 6. No ground-truth path is available to confirm absolute accuracy however it can be seen that the corrected receiver (purple) does not exhibit any instability or other bad behaviour compared to the uncorrected receiver (green).

\section{CONCLusion And Future Work}

A Differential GPS system was implemented using only low-cost GPS receivers and antennae and was tested in static and roving environments. It was found to perform badly in high multipath environments such as urban areas due to the nature of the low-cost receivers and antennae used.

In open environments, it was found to produce an error in altitude of between one and four metres, however the altitude error is less important for field robotics than horizontal as other sources of information are available on the vertical axis such as barometric altitude.

The error in the East/North plane with respect to ground truth was found to be reliably on the order of one metre making it suitable for multi-agent tasks such as formation control and inter-agent collision avoidance. It was common for the accuracy of the system to be better along the East axis than the North due to a poor estimate of receiver clock error at the reference station combining with the geometry of the satellites.

Future work would include the profiling of the clock in the reference receiver in order to find an optimal estimator for that clock error. This would in turn allow more consistant accuracy along all axes. The accuracy of this DGPS system with respect to inter-agent metrics in a multi-agent system will also be investigated.

Given the low cost of the reference receivers, multiple such receivers may be distributed in the experimental area and a consensus reached on the corrections that should be largely free of error from any one receiver clock. The positional solutions would also benefit from integration with other sources of data such as the received signal strength of inter-agent communications. This would provide a largely independent estimate of agent relative positions which in turn could be used to improve the estimates of agent location and, indirectly, improve the accuracy of the GPS corrections as well.

\section{ACKNOWLEDGEMENTS}

This work was in part supported by a joint project between ANU and Shandong Academy of Sciences. C. Yu was supported by the Australian Research Council through a Queen Elizabeth II Fellowship and Discovery Projects DP110100538 and DP-130103610, and the Overseas Expert Program of Shandong Province.

\section{REFERENCES}

[1] E. D. Kaplan and C. J. Hegarty, Understanding GPS: Principles and Applications, Second Edition. Artech House, 2006.

[2] L. Jurisica, F. Duchon, D. Kastan, and A. Babinec, "High Precision GNSS Guidance for Field Mobile Robots," International Journal of Advanced Robotic Systems, p. 1, 2012.

[3] K. Ohno, T. Tsubouchi, B. Shigematsu, S. Maeyama, and S. Yuta, "Outdoor navigation of a mobile robot between buildings based on DGPS and odometry data fusion," in IEEE Conference on Robotics and Automation, Taipei, Taiwan, 2003, pp. 1978-1984.

[4] B. Wu, T. Lee, and H. Chang, "GPS navigation based autonomous driving system design for intelligent vehicles," in IEEE International Conference on Systems, Man and Cybernetics, Montreal, 2007, pp. 3294-3299.

[5] M. S. Grewal, L. R. Weill, and A. P. Andrews, Global Positioning Systems, Inertial Navigation, and Integration. Wiley, 2007.

[6] H. Carvalho and P. D. Moral, "Optimal nonlinear filtering in GPS/INS integration," IEEE Transactions on Aerospace and Electronic Systems, vol. 33, no. 3, pp. 835-850, 1997.

[7] A. English, D. Ball, P. Ross, B. Upcroft, G. Wyeth, and P. Corke, "Low Cost Localisation for Agricultural Robotics," in Australasian Conference on Robotics and Automation, 2013.

[8] R. Lenain, B. Thuilot, C. Cariou, and P. Martinet, "Adaptive and predictive non linear control for sliding vehicle guidance," in IEEE/RSJ International Conference on Intelligent Robots and Systems, Sendai, 2004, pp. 455-460.

[9] C. Low and D. Wang, "GPS-based path following control for a car-like wheeled mobile robot with skidding and slipping," IEEE Transactions on Control Systems Technology, vol. 16, no. 2, pp. 340-347, 2008.

[10] T. Takasu and A. Yasuda, "Development of the low-cost RTK-GPS receiver with an open source program package RTKLIB," in International Symposium on GPS/GNSS, International Convention Center Jeju, Korea, 2009.

[11] D. Gebre-Egziabher and G. H. Elkaim, "MAV attitude determination by vector matching," IEEE Transactions on Aerospace and Electronic Systems, vol. 44, no. 3, pp. 1012-1028, July 2008.

[12] J. C. Cohenour, "Global Positioning System Clock and Orbit Statistics and Precise Point Positioning," Ph.D. dissertation, Ohio University, 2009.

[13] R. Hatch., "Synergism of GPS code and carrier measurements," in 3rd International Geodetic Sypomsium on Satellite Doppler Positioning, Las Cruces, New Mexico, 1982, pp. Vol. 2 1213-1232.

[14] Global Positioning Systems Directorate, Systems Engineering and Integration Interface Specification IS-GPS-200: Navstar GPS Space Segment/Navigation User Interfaces, 2013.

[15] RTCM Special Committee No. 104, "RTCM 10402.3 Recommended Standards for Differential GPS," 2010.

[16] RTCM Special Committee No. 104, "RTCM Standard 10403.2 Differential GNSS Services Version 3," 2013.

[17] U-blox AG, "u-Blox LEA-6," 2013. [Online]. Available: http://www. u-blox.com/en/gps-modules/u-blox-6-timing-module/lea-6t.html

[18] Canadian Spatial Reference System, "Online Global GPS Processing Service,” 2013. [Online]. Available: http://www.geod.nrcan.gc.ca/ 